Supplementary Information for

\title{
Imaging Dual-Moiré Lattices in Twisted Bilayer Graphene Aligned on Hexagonal Boron Nitride Using Microwave Impedance Microscopy
}

Authors: Xiong Huang ${ }^{1,2 \dagger}$, Lingxiu Chen ${ }^{3 \dagger}$, Shujie Tang ${ }^{4}$, Chengxin Jiang ${ }^{5}$, Chen Chen ${ }^{5}$, Huishan Wang ${ }^{5}$, Zhi-Xun Shen ${ }^{6}$, Haomin Wang ${ }^{5}$, Yong-Tao Cui ${ }^{1 *}$

\author{
Affiliations: \\ ${ }^{1}$ Department of Physics and Astronomy, University of California, Riverside, California, 92521, \\ USA \\ ${ }^{2}$ Department of Materials Science and Engineering, University of California, Riverside, \\ California, 92521, USA \\ ${ }^{3}$ School of Materials Science and Physics, China University of Mining and Technology, \\ Xuzhou, 221116, China \\ ${ }^{4} 2020$ X-Lab, Shanghai Institute of Microsystem and Information Technology, Chinese \\ Academy of Sciences, Shanghai 200050, China \\ ${ }^{5}$ State Key Laboratory of Functional Materials for Informatics, Shanghai Institute of \\ Microsystem and Information Technology, Chinese Academy of Sciences, Shanghai 200050, \\ China \\ ${ }^{6}$ Department of Physics and Applied Physics, Geballe Laboratory for Advanced Materials, \\ Stanford University, Stanford, CA 94305, USA \\ $\dagger$ These authors contribute equally to this work. \\ *Correspondence to: yongtao.cui@ucr.edu
}

S1. MIM effective circuit models and response curves

S2. Data on additional samples

S3. Atomic stacking model for dual moiré structures

S4. FFT analysis of dual-moiré patterns 


\section{S1. MIM effective circuit models and response curves}

In the conventional model for the tip-sample impedance, the tip couples to the sample capacitively via an interface capacitor that accounts for the insulating spacer. The typical response curves are plotted in Fig. S1a. The signal difference between the insulating and conductive limits of sample conductivity provides a reference for the maximum MIM-Im contrast one can expect for this model. In typical experiments, a calibration sample, such as patterned Al thin film which has a native oxide layer (a couple of nanometers thick) on the surface, can be used to obtain a reference value for the maximum MIM-Im contrast. In our MIM measurement on graphene moiré systems, the signal contrast between center and corner of the moire hexagon is often much larger than the maximum reference contrast measured on the calibration sample, indicating the breakdown of the conventional tip-sample impedance model.

As suggested by our combined MIM-cAFM measurement, the tip-sample interface has a contact resistance that is on the order of 10's of kOhms. The effective circuit model and the corresponding response curves are shown in Fig. S1b. Note that the span of the tip-sample admittance is much larger than that of the conventional model. The MIM signal contrast of the moiré pattern from Fig. $1 \mathrm{~b}$ in the main text, after calibration of the signal gain, corresponds to an admittance change of $\sim 6$ $\mu \mathrm{S}$, which agrees reasonably well with estimates from cAFM data in Fig. 1c, $\sim 2 \mu \mathrm{S}$. The deviation could be due to the uncertainty in the tip geometry used in the calibration procedure.
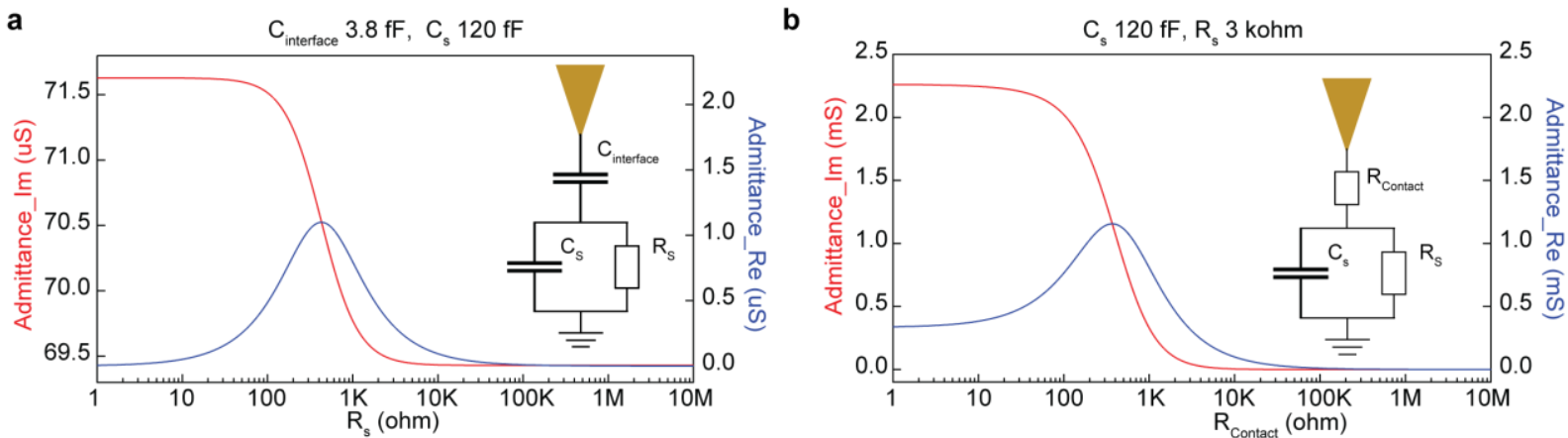

Figure S1. Simulated MIM response curves based on two effective circuit models for the tipsample interaction. (a) The tip-sample contact does not have a good electrical conduction and is thus dominated by an interface capacitance. This is the case for MIM measurements on most of common samples. The response curves plot the imaginary and real parts of the tip-sample admittance as a function of varying sample resistance. (b) The tip-sample contact has a good electrical conduction which can be modelled by a contact resistance. This is the case for imaging moiré patterns in graphene/hBN samples in this work. The response curves plot the imaginary and real parts of the tip-sample admittance (inverse of impedance) as a function of varying tip-sample contact resistance. 
S2. Data on additional samples


Figure S2. MIM/cAFM images for TBG/hBN samples D2-D5 (a, c, e and g) and their FFT images (b, d, f and h). All scale bars: $15 \mathrm{~nm}$. 


\section{S3. Atomic stacking model for dual-moiré structures}

The periodic atomic lattice for each of the three layers in a TBG/hBN structure can be described by the lowest harmonics:

$$
f(\vec{r})=\sum_{i=1}^{3} e^{i \vec{k}_{i} \cdot \vec{r}}
$$

where $\vec{k}_{i}(i=1,2,3)$ are the unit vectors for individual atomic lattice. Thus $\vec{k}_{i} \cdot \vec{r}$ represent the phase of the lattice. The formation of moiré patterns depends on the alignment among the phases of the three layers. The center of each moiré-S domain can be identified by the location where the three lattice phases are closest to each other (See an example in Fig. S3a). We thus define a phase variation as

$$
\begin{aligned}
\Delta_{\text {phase }}(\vec{r})= & \left(\operatorname{wrap}\left(\vec{k}_{1}^{h B N} \cdot \vec{r}-\vec{k}_{1}^{G 1} \cdot \vec{r}\right)\right)^{2}+\left(\operatorname{wrap}\left(\vec{k}_{2}^{h B N} \cdot \vec{r}-\vec{k}_{2}^{G 1} \cdot \vec{r}\right)\right)^{2} \\
& +\left(\operatorname{wrap}\left(\vec{k}_{1}^{h B N} \cdot \vec{r}-\vec{k}_{1}^{G 2} \cdot \vec{r}\right)\right)^{2}+\left(\operatorname{wrap}\left(\vec{k}_{2}^{h B N} \cdot \vec{r}-\vec{k}_{2}^{G 2} \cdot \vec{r}\right)\right)^{2} \\
& +\left(\operatorname{wrap}\left(\vec{k}_{1}^{G 1} \cdot \vec{r}-\vec{k}_{1}^{G 2} \cdot \vec{r}\right)\right)^{2}+\left(\operatorname{wrap}\left(\vec{k}_{2}^{G 1} \cdot \vec{r}-\vec{k}_{2}^{G 2} \cdot \vec{r}\right)\right)^{2}
\end{aligned}
$$

Here the wrap() function wraps the phase value to the range $[-\pi, \pi]$. The centers of moiré-S domains correspond to the minimum values of $\Delta_{\text {phase }}$. To find the analytical expressions for the unit vectors, we find the center locations of adjacent moiré-S domains in the following way. First, it is obvious that $\vec{r}=0$ corresponds to the minimum location of $\Delta_{\text {phase }}$ in the $(0,0)$ moire-S domain around origin. Second, we solve the local minimum locations of $\Delta_{\text {phase }}$ in the $(1,0)$ and $(0,1)$ moiré-S domains, respectively. For simplicity, we define $\Delta \vec{k}_{i}^{a}=\vec{k}_{i}^{h B N}-\vec{k}_{i}^{G 1}, \Delta \vec{k}_{i}^{b}=$ $\vec{k}_{i}^{h B N}-\vec{k}_{i}^{G 2}$ and $\Delta \vec{k}_{i}^{c}=\vec{k}_{i}^{G 1}-\vec{k}_{i}^{G 2}$, where $i=1$ or 2.

In the $(1,0)$ domain, there should be an accumulated phase difference of $2 \pi$ between G1 and G2 as well as between hBN and G2 along $\Delta \vec{k}_{1}^{b}$ and $\Delta \vec{k}_{1}^{c}$. Therefore, we can manually add the phase difference to remove the $\operatorname{wrap}()$ function and we get:

$$
\begin{gathered}
\Delta_{\text {phase in }(1,0)}(\vec{r})=\left(\Delta \vec{k}_{1}^{a} \cdot \vec{r}\right)^{2}+\left(\Delta \vec{k}_{2}^{a} \cdot \vec{r}\right)^{2}+\left(\Delta \vec{k}_{1}^{b} \cdot \vec{r}-2 \pi\right)^{2}+\left(\Delta \vec{k}_{2}^{b} \cdot \vec{r}\right)^{2} \\
+\left(\Delta \vec{k}_{1}^{c} \cdot \vec{r}-2 \pi\right)^{2}+\left(\Delta \vec{k}_{2}^{c} \cdot \vec{r}\right)^{2}
\end{gathered}
$$

The local minimum location can be obtained by solving $\frac{\partial}{\partial x} \Delta_{\text {phase }}(\vec{r})=0$ and $\frac{\partial}{\partial y} \Delta_{\text {phase }}(\vec{r})=0$, which gives

where $i, j=1$ or $2, m, n=a, b$, or $c$.

$$
\vec{r}_{1}=\frac{2 \pi \sum_{i, m}\left[\left(\Delta \vec{k}_{1}^{b}+\Delta \vec{k}_{1}^{c}\right) \cdot\left(\Delta \vec{k}_{i}^{m} \times \hat{z}\right)\right]\left(\Delta \vec{k}_{i}^{m} \times \hat{z}\right)}{\frac{1}{2} \sum_{i, j, m, n}\left|\Delta \vec{k}_{i}^{m} \times \Delta \vec{k}_{j}^{n}\right|^{2}}
$$

Similarly, in the $(0,1)$ domain, the additional term of $2 \pi$ should be added to $\Delta \vec{k}_{2}^{b} \cdot \vec{r}$ and $\Delta \vec{k}_{2}^{c} \cdot \vec{r}$. The local minimum location is

where $i, j=1$ or $2, m, n=a, b$, or $c$.

$$
\vec{r}_{2}=\frac{2 \pi \sum_{i, m}\left[\left(\Delta \vec{k}_{2}^{b}+\Delta \vec{k}_{2}^{c}\right) \cdot\left(\Delta \vec{k}_{i}^{m} \times \hat{z}\right)\right]\left(\Delta \vec{k}_{i}^{m} \times \hat{z}\right)}{\frac{1}{2} \sum_{i, j, l, m}\left|\Delta \vec{k}_{i}^{m} \times \Delta \vec{k}_{j}^{n}\right|^{2}}
$$

The other unit vector is then $\vec{r}_{3}=\vec{r}_{2}-\vec{r}_{1}$. 
Note that if we pick the hBN layer to be identical as the G1 layer, the trilayer stack should become a G1/G2 bilayer stack. Indeed, by setting $\vec{k}_{i}^{h B N}=\vec{k}_{i}^{G 1}$ hence $\Delta \vec{k}_{i}^{a}=0$ and $\Delta \vec{k}_{i}^{b}=\Delta \vec{k}_{i}^{c}$, it can be verified that the analytical expressions for $\vec{r}_{1}$ and $\vec{r}_{2}$ reduce to those expected for a bilayer stack.

As another sanity check, Fig. S3b plots the calculated $\Delta_{\text {phase }}$ for the case in Fig. S3a. The markers indicate the locations of the minimum values of $\Delta_{\text {phase }}$, which match well the atomic patterns in Fig. S3a.
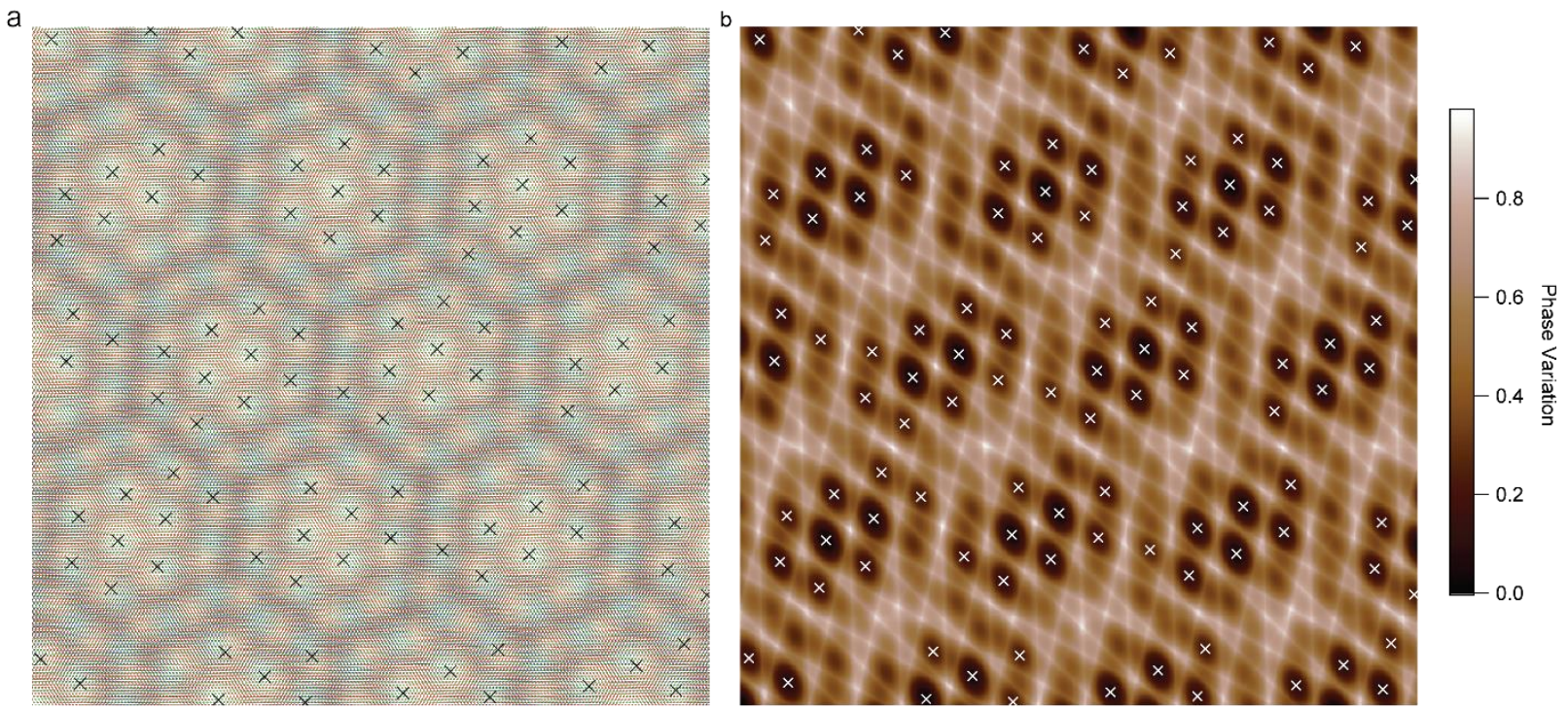

Figure S3. (a) Atomic stacking model for twisted bilayer graphene $\left(3.6^{\circ}\right)$ on $\mathrm{hBN}$. Blue atoms for $\mathrm{hBN}$, red atoms for aligned graphene and green atoms for rotated graphene, respectively. (b) Calculated phase variation $\Delta_{\text {phase }}$. Locations of the minimum values of $\Delta_{\text {phase }}$ are indicated by the markers in both (a) and (b). 


\section{S4. FFT analysis on dual-moiré patterns}

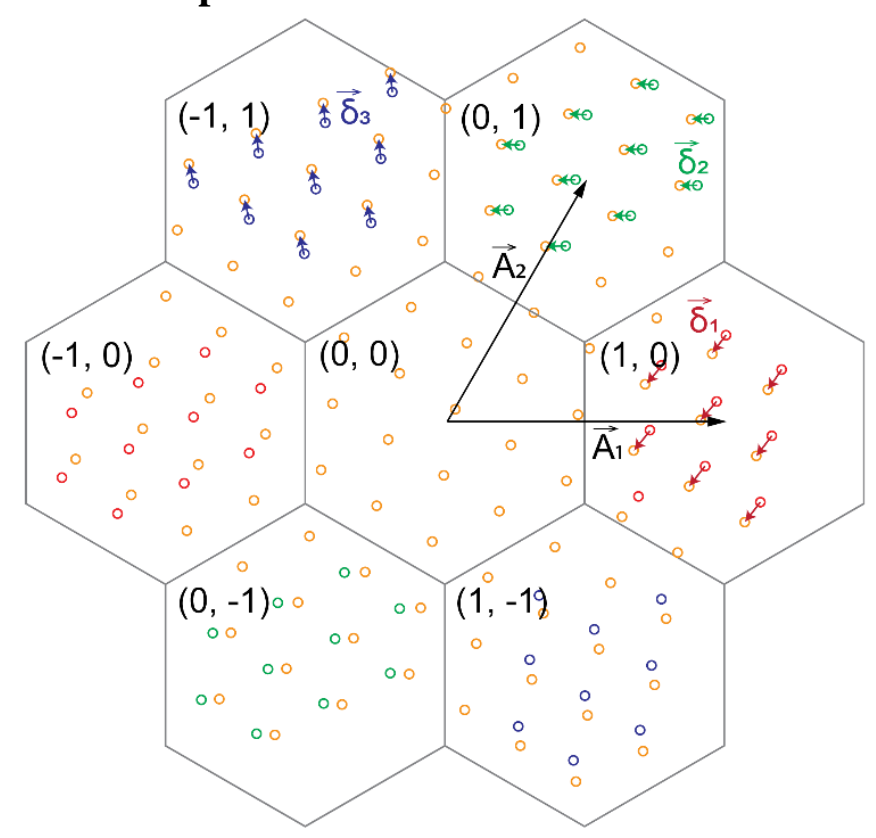

Figure S4. FFT analysis of a periodic pattern with discontinuities at the boundaries of the large periodic structure. Orange circles are the lattice extended from domain $(0,0)$ without including any discontinuities. Red circles are those for hexagon domains $(1,0)$ and $(-1,0)$. Green (blue) ones are for domains $(0,1)$ and $(0,-1)((-1,1)$ and $(1,-1))$.

In this section, we present the mathematical analysis on the Fourier transform of a dual moiré superstructure in which the moiré-S pattern has discontinuities at the boundaries of the moiré-L unit cell domains.

The periodic structure of the triangular moiré-S lattice can be described by the lowest harmonics of the following form:

$$
f(\vec{r})=\sum_{i=1}^{3} e^{i \vec{k}_{i} \cdot \vec{r}}
$$

with $\vec{k}_{1}, \vec{k}_{2}$ and $\vec{k}_{3}=\vec{k}_{2}-\vec{k}_{1}$ the three unit vectors of the moiré-S lattice.

Across the boundaries of the moiré-L domains, the discontinuities in the moiré-S lattice can be modelled as two displacement vectors, $\vec{\delta}_{1}$ and $\vec{\delta}_{2}$, along the two directions defined by the two unit vectors of the moiré-L lattice, $\vec{A}_{1}$ and $\vec{A}_{2}$, respectively. In the (m, n)-th unit cell of the moiré-L lattice, as shown in Fig. S4, the overall accumulated displacement will be:

$$
\vec{\delta}_{m n}=m \vec{\delta}_{1}+n \vec{\delta}_{2}
$$

Therefore, in general, the moiré-S lattice with discontinuities can be expressed as

$$
f(\vec{r})=\sum_{i=1}^{3} e^{i \vec{k}_{i} \cdot\left(\vec{r}-\vec{\delta}_{m n}\right)}
$$


for $\vec{r}$ within the (m, n)-th domain. Therefore, the Fourier transform of $f(\vec{r})$ can be obtained by calculating the Fourier transform of each term, $F_{i}(\vec{k})$. Thus, we have:

where the integral is over the entire plane.

$$
F_{i}(\vec{k})=\iint e^{i \vec{k}_{i} \cdot\left(\vec{r}-\vec{\delta}_{m n}\right)} \cdot e^{-i \vec{k} \cdot \vec{r}} d^{2} \vec{r}
$$

We evaluate the integral by summing the contribution from individual unit cells. Specifically, in the (m, n)-th unit cell which is centered at $\vec{R}_{m, n}=m \cdot \vec{A}_{1}+n \cdot \vec{A}_{2}$, we define a reduced position vector $\vec{r}_{0}=\vec{r}-\vec{R}_{m, n}$, and we express the integral in terms of $\vec{r}_{0}$.

$$
\begin{gathered}
F_{i}(\vec{k})=\sum_{m, n} F_{i}^{m n}(\vec{k})=\sum_{m, n} \iint_{u . c .} e^{i \vec{k}_{i} \cdot\left(\vec{r}_{0}+\vec{R}_{m, n}-m \cdot \vec{\delta}_{1}-n \cdot \vec{\delta}_{2}\right)} \cdot e^{-i \vec{k} \cdot\left(\vec{r}_{0}+\vec{R}_{m, n}\right)} d^{2} \vec{r}_{0} \\
=\sum_{m, n} e^{i \vec{k}_{i} \cdot\left(\vec{R}_{m, n}-m \cdot \vec{\delta}_{1}-n \cdot \vec{\delta}_{2}\right)-i \vec{k} \cdot \vec{R}_{m, n}} \iint_{u . c .} e^{i\left(\vec{k}_{i}-\vec{k}\right) \cdot \vec{r}_{0}} d^{2} \vec{r}_{0} \\
=\sum_{m, n} e^{i \vec{k}_{i} \cdot\left(\vec{R}_{m, n}-m \cdot \vec{\delta}_{1}-n \cdot \vec{\delta}_{2}\right)-i \vec{k} \cdot \vec{R}_{m, n}} F_{i}^{00}(\vec{k})
\end{gathered}
$$

Note that $F_{i}^{00}(\vec{k}) \equiv \iint_{\text {u.c. }} e^{i\left(\vec{k}_{i}-\vec{k}\right) \cdot \vec{r}_{0}} d^{2} \vec{r}_{0}$ is simply the integral within the $(0,0)$-th unit cell which is a constant independent of $m$ and $n$. We then have

$$
\begin{aligned}
& F_{i}(\vec{k})=F_{i}^{00}(\vec{k}) \sum_{m, n} e^{i \vec{k}_{i} \cdot\left(\vec{R}_{m, n}-m \cdot \vec{\delta}_{1}-n \cdot \vec{\delta}_{2}\right)-i \vec{k} \cdot \vec{R}_{m, n}} \\
& =F_{i}^{00}(\vec{k}) \sum_{m, n} e^{i\left[\left(\vec{k}_{i}-\vec{k}\right) \cdot\left(m \cdot \vec{A}_{1}+n \cdot \vec{A}_{2}\right)-m \vec{k}_{i} \cdot \vec{\delta}_{1}-n \vec{k}_{i} \cdot \vec{\delta}_{2}\right]} \\
& =F_{i}^{00}(\vec{k}) \sum_{m}^{m, n} e^{i m \cdot\left(\left(\vec{k}_{i}-\vec{k}\right) \cdot \vec{A}_{1}-\vec{k}_{i} \cdot \vec{\delta}_{1}\right)} \sum_{n} e^{i n \cdot\left(\left(\vec{k}_{i}-\vec{k}\right) \cdot \vec{A}_{2}-\vec{k}_{i} \cdot \vec{\delta}_{2}\right)} \\
& =F_{i}^{00}(\vec{k}) \frac{1-e^{i M\left[\left(\vec{k}_{i}-\vec{k}\right) \cdot \vec{A}_{1}-\vec{k}_{i} \cdot \vec{\delta}_{1}\right]}}{1-e^{i\left[\left(\vec{k}_{i}-\vec{k}\right) \cdot \vec{A}_{1}-\vec{k}_{i} \cdot \vec{\delta}_{1}\right]}} \frac{1-e^{i N\left[\left(\vec{k}_{i}-\vec{k}\right) \cdot \vec{A}_{2}-\vec{k}_{i} \cdot \vec{\delta}_{2}\right]}}{1-e^{i\left[\left(\vec{k}_{i}-\vec{k}\right) \cdot \vec{A}_{2}-\vec{k}_{i} \cdot \vec{\delta}_{2}\right]}} \\
& \left|F_{i}(\vec{k})\right|=\frac{\sin \left(\frac{M}{2}\left[\left(\vec{k}_{i}-\vec{k}\right) \cdot \vec{A}_{1}-\vec{k}_{i} \cdot \vec{\delta}_{1}\right]\right)}{\sin \left(\frac{1}{2}\left[\left(\vec{k}_{i}-\vec{k}\right) \cdot \vec{A}_{1}-\vec{k}_{i} \cdot \vec{\delta}_{1}\right]\right)} \cdot \frac{\sin \left(\frac{N}{2}\left[\left(\vec{k}_{i}-\vec{k}\right) \cdot \vec{A}_{2}-\vec{k}_{i} \cdot \vec{\delta}_{2}\right]\right)}{\sin \left(\frac{1}{2}\left[\left(\vec{k}_{i}-\vec{k}\right) \cdot \vec{A}_{2}-\vec{k}_{i} \cdot \vec{\delta}_{2}\right]\right)} F_{i}^{00}(\vec{k})
\end{aligned}
$$

The peak positions of $\left|F_{i}(\vec{k})\right|$, denoted as $\vec{k}_{i}^{\prime}$, satisfy the conditions:

$$
\left(\vec{k}_{i}-\vec{k}_{i}^{\prime}\right) \cdot \vec{A}_{1}-\vec{k}_{i} \cdot \vec{\delta}_{1}=0 \&\left(\vec{k}_{i}-\vec{k}_{i}^{\prime}\right) \cdot \vec{A}_{2}-\vec{k}_{i} \cdot \vec{\delta}_{2}=0
$$

Define the reciprocal vectors $\vec{k}_{A 1}$ and $\vec{k}_{A 2}$ as

$$
\begin{aligned}
& \vec{k}_{A 1}=\frac{\vec{A}_{2} \times \vec{e}_{z}}{\left|\vec{A}_{1} \times \vec{A}_{2}\right|} \\
& \vec{k}_{A 2}=\frac{\vec{e}_{z} \times \vec{A}_{1}}{\left|\vec{A}_{1} \times \vec{A}_{2}\right|}
\end{aligned}
$$

And they satisfy the relations $\vec{k}_{A 1} \cdot \vec{A}_{1}=1$ and $\vec{k}_{A 2} \cdot \vec{A}_{2}=1$. We can then obtain 


$$
\vec{k}_{i}-\vec{k}_{i}^{\prime}=\vec{k}_{A 1} \cdot\left(\vec{k}_{i} \cdot \vec{\delta}_{1}\right)+\vec{k}_{A 2} \cdot\left(\vec{k}_{i} \cdot \vec{\delta}_{2}\right)
$$

or

$$
\vec{k}_{i}^{\prime}=\vec{k}_{i}-\left[\vec{k}_{A 1} \cdot\left(\vec{k}_{i} \cdot \vec{\delta}_{1}\right)-\vec{k}_{A 2} \cdot\left(\vec{k}_{i} \cdot \vec{\delta}_{2}\right)\right]
$$

We can see that the Fourier peak positions of the discontinuous lattice, $\vec{k}_{i}^{\prime}$, are shifted from those for a continuous lattice, $\vec{k}_{i}$, by a vector that depends on the displacement vectors, $\vec{\delta}_{1}$ and $\vec{\delta}_{2}$, and the reciprocal vectors corresponding to the moiré-L lattice, $\vec{k}_{A 1}$ and $\vec{k}_{A 2}$.

Experimentally, we can find $\vec{k}_{i}^{\prime}$ from the Fourier transform of the entire image containing large number of moiré-L unit cells, and $\vec{k}_{i}$ can be determined from the Fourier transform of the data within individual moiré-L unit cells. $\vec{k}_{A 1}$ and $\vec{k}_{A 2}$ can also be determined by extracting the unit vectors for the moiré-L lattice. From these, we can then determine the displacement vectors, $\vec{\delta}_{1}$ and $\vec{\delta}_{2}$. Alternatively, $\vec{\delta}_{1}$ and $\vec{\delta}_{2}$ can also be determined by comparing the moiré-S lattice patterns in neighboring moiré-L unit cells. The results from these two methods match very well. 